\title{
Effects of the balance between type 1 and type 2 T helper cells on ovarian cancer
}

\author{
C.J. Hao ${ }^{1}$, J. Li ${ }^{2}$, P. Liu ${ }^{1}$, X.L. Li ${ }^{1}$, Y.Q. Hu ${ }^{1}$, J.C. Sun ${ }^{3}$ and Y. Wei ${ }^{4}$ \\ ${ }^{1}$ Obstetrics and Gynecology, \\ Panyu Maternal and Child Care Service Centre of Guangzhou, Guangzhou, \\ Guangdong, China \\ ${ }^{2}$ Obstetrics and Gynecology, General Hospital of the Guangzhou Military Region, \\ Guangzhou, Guangdong, China \\ ${ }^{3}$ Department of Radiotherapy, \\ the First Affiliated Hospital of Guangzhou Medical University, \\ Guangzhou, Guangdong, China \\ ${ }^{4}$ Intensive Care Unit, Guangdong No. 2 Provincial People's Hospital, \\ Guangzhou, Guangdong, China \\ Corresponding authors: J.C. Sun / Y. Wei \\ E-mail: sunjiancc_12@163.com / wei_yicn353@163.com
}

Genet. Mol. Res. 15 (2): gmr.15027936

Received October 28, 2015

Accepted February 12, 2016

Published June 3, 2016

DOI http://dx.doi.org/10.4238/gmr.15027936

\begin{abstract}
The balance between type 1 and type $2 \mathrm{~T}$ helper cells (the Th1-Th2 balance) is closely correlated with cancer, but the correlation in ovarian cancer remains unconfirmed. We investigated the Th1-Th2 balance for the diagnosis, treatment, and prognostic evaluation of ovarian cancer. Fifty healthy subjects and 50 ovarian cancer patients were recruited. The levels of various cytokines were determined in sera and ovarian cancer tissues using a Th1-Th2 human cytokine array. The usefulness of TNF $\alpha$, IFN $\gamma, \mathrm{TNF} \alpha / \mathrm{IL}-4$, and IFN $\gamma / \mathrm{IL}-4$ for ovarian cancer diagnosis was assessed based on receiver operating characteristic (ROC) curves. The relationship between the TNF $\alpha / \mathrm{IL}-4$ level and survival time was investigated based on a survival curve. In the ovarian cancer patients, the levels of Th1 factors (IL-2, IFN $\gamma$,
\end{abstract}


TNF $\alpha$, and IL-13) increased significantly in the sera, and IFN $\gamma$ and $\mathrm{TNF} \alpha$ increased significantly in the ovarian cancer tissues. The levels of Th2 factors (IL-5 and IL-6) increased in the sera, but the level of IL-6 decreased significantly in the ovarian cancer tissues. Serum TNF $\alpha / \mathrm{IL}-4$ and IFN $\gamma /$ IL-4 levels increased significantly in the peripheral blood of the ovarian cancer patients. ROC curve analysis revealed that TNF $\alpha$, IFN $\gamma, \mathrm{TNF} \alpha / \mathrm{IL}-4$, and IFN $\gamma / \mathrm{IL}-4$ levels are useful for ovarian cancer diagnosis, with area under the curve values of $0.831,0.753,0.846$, and 0.803 , respectively. The TNF $\alpha / \mathrm{IL}-4$ level in the ovarian cancer patients was positively correlated with survival time, and the Th1-Th2 balance shifted toward Th1 in the ovarian cancer patients. The TNF $\alpha / \mathrm{IL}-4$ ratio might be useful for the diagnosis and prognosis of ovarian cancer.

Key words: Ovarian cancer; Th1-Th2 balance; Survival curve; Cytokine array

\section{INTRODUCTION}

A shift in the balance between type 1 and type $2 \mathrm{~T}$ helper cells (Th1 and Th2 cells) is considered an important factor in cancer development (Shurin et al., 1999; Chen et al., 2004; Green et al., 2010). Th1 cells mainly secrete IL-2, TNF $\alpha$, IFN $\gamma$, and other cytokines to promote cell immunity (Mills et al., 1993), thereby playing an important role in anti-viral (Zhang et al., 2014) and anti-tumor activity. Th2 cells mainly secrete IL-4, IL-5, IL-6, IL-10, and other cytokines to induce humoral immunity, thereby playing an important role in foreign antigen clearance (Kidd, 2003). Th1 and Th2 check and balance each other in the body. Many studies have indicated that Th1-Th2 imbalance allows tumor cells to escape immune surveillance and clearance; thus, a shift in the Th1-Th2 balance is an important factor in the development and progression of tumors (Clerici et al., 1996; Jones et al., 2002). Th1-Th2 imbalance has been observed in a variety of cancers, and has been used to assess tumor progression and prognosis in clinical practice (Wada et al., 2003; Zhao et al., 2008; Block et al., 2011).

Ovarian cancer represents approximately $3 \%$ of cancers in women but causes more deaths than any other cancer of the female reproductive system (Chornokur et al., 2013). The role of the Th1-Th2 balance in ovarian cancer has been described. Aoki et al. (2000) used flow cytometry to investigate changes in the Th1-Th2 balance after chemotherapy and granulocytecolony stimulating factor (G-CSF) injection in ovarian cancer patients, and determined that the balance shifted toward Th2 10 days after chemotherapy, and toward Th1 after G-CSF treatment. However, these authors did not determine the Th1-Th2 balance before treatment. The immune system is greatly affected by cancer treatment; therefore, their findings do not reflect the Th1Th2 status before treatment. Kusuda et al. (2005) determined the mRNA levels of TNF $\alpha$, IFN $\gamma$, and IL-12p40 in Th1 cells, and those of IL- 6 and IL-10 in Th2 cells in ovarian cancer tissues by quantitative polymerase chain reaction. They thereby determined the correlations between those mRNA levels and the clinical stage, histological grade, and histological type of ovarian cancer. The authors concluded that the expression levels of IFN $\gamma$, IL-12p40, and IL-6 might represent useful prognostic molecular markers for patients with advanced ovarian cancer, but did not investigate whether the absolute level of these markers or the Th1-Th2 balance in peripheral blood was relevant to the disease. Zhu et al. (2010) investigated the levels of IFN 
TNF $\alpha$, IL-10, IL-5, IL-4, and IL-2 before surgery for ovarian cancer using cytokine arrays, and found that IL-2 and IL-10 levels are paired predictors of survival. However, these authors did not investigate alterations in Th1-Th2 levels in cancer tissue.

In this study, the levels of relevant cytokines were quantitatively detected in the sera and cancer tissues from ovarian cancer patients using Th1-Th2 human cytokine arrays, and the usefulness of these measurements for the diagnosis and prognosis of ovarian cancer was investigated.

\section{MATERIAL AND METHODS}

\section{Patients}

Fifty ovarian cancer patients who received treatment at the Panyu Maternal and Child Care Service Centre and at the First Affiliated Hospital of Guangzhou Medical University between 2012 and 2014 were recruited. The New International Ovarian Cancer Diagnostic Criteria were used to diagnose and grade ovarian cancer (Sugiyama and Kou, 2008; Höhn et al., 2014). Patients were diagnosed for the first time with ovarian cancer and they had not received any drug or surgery treatment before the serum samples were collected. Ovarian cancer tissues and corresponding adjacent tissues or tissue from the opposite ovary were separated under a microscope after surgery and immediately frozen at $-80^{\circ} \mathrm{C}$.

Fifty healthy subjects undergoing health examinations in the same hospital were selected as controls. The healthy subjects were age-matched with the patients and had no history of gynecological disease.

This study was approved by the Ethics Committee of Guangzhou Medical University. Informed consent was obtained from all patients on the day of admission.

\section{Protein extraction from cancer tissue}

Total protein was extracted from tumor tissue using radio-immunoprecipitation assay (RIPA) buffer (Solarbio Biotech Corp., Beijing, China) following the manufacturer instructions. Briefly, ovarian cancer tissues were lysed for use in antibody array analysis in RIPA buffer containing 1\% phenylmethylsulfonyl fluoride (Solarbio Biotech Corp.), and homogenized using a microtissue grinder (Kimble Chase, Thailand). The supernatants were collected after centrifugation at $14,000 \mathrm{~g}$ and $4^{\circ} \mathrm{C}$ for $15 \mathrm{~min}$. The protein concentration was determined using a bicinchoninic acid assay (Solarbio Biotech Corp.).

\section{Serum cytokine profile obtained using a human cytokine microarray}

A commercial quantitative microarray (Th1-Th2 Human Cytokine Array 1, BD, USA) was used to obtain the cytokine expression profile of the serum samples according to the manufacturer instructions, and the results were analyzed using the RayBiotech Q-Analyzer program. Briefly, after a 30-min incubation with blocking buffer, the glass chips were washed, and each well was overlaid with $100 \mu \mathrm{L}$ diluted sample. After overnight incubation at $4^{\circ} \mathrm{C}$ and extensive washing, a biotin-labeled detection antibody was added and left for $1 \mathrm{~h}$. The chips were then rinsed and incubated with Alexa Fluor 555-conjugated streptavidin for $1 \mathrm{~h}$ at room temperature. The fluorescence was then recorded (555 nm excitation and $655 \mathrm{~nm}$ emission) using a GenePix 4000B laser scanner (Axon Instruments, Foster City, CA, USA). 


\section{Statistical analysis}

All results and relative Th1-Th2 ratios are reported as means \pm SE. Statistical analysis was performed using the SPSS 13.0 software. The paired Student $t$-test was used to compare groups. Significance was tested using the homogeneity test $(\mathrm{P}<0.05$ was considered significant $)$.

\section{RESULTS}

\section{Expression of Th1-Th2 in healthy subjects and in the serum, cancer tissue, and adjacent tissue of ovarian cancer patients}

A Th1-Th2 human cytokine array was used to quantitatively detect the expression of Th1 factors (IL-2, IL-13, IFN $\gamma$, and TNF $\alpha$ ) and Th2 factors (IL-4, IL-5, IL-6, and IL-10) in the peripheral blood of healthy individuals and patients with ovarian cancer before treatment, as well as in ovarian cancer tissues and adjacent tissues. The results are shown in Figure 1 and Table 1. Compared with the healthy controls, serum Th1 cytokine levels were generally increased in the ovarian cancer patients as follows: IL-2 was increased 1.42-fold (paired $t$-test; $\mathrm{P}=0.023 ; \mathrm{N}=50$ for each group); IL-13 was increased 1.73-fold (paired $t$-test; $\mathrm{P}=0.012 ; \mathrm{N}$ $=50$ for each group); IFN $\gamma$ was increased 1.52 -fold (paired $t$-test; $\mathrm{P}=0.045 ; \mathrm{N}=50$ for each group); and TNF $\alpha$ was increased 1.91-fold (paired $t$-test; $\mathrm{P}=0.013 ; \mathrm{N}=50$ for each group). Th2 cytokines were generally increased in patients with ovarian cancer as follows: IL-6 was increased 2.56-fold (paired $t$-test; $\mathrm{P}=0.014 ; \mathrm{N}=50$ for each group), and IL-5 was increased 2.41 -fold (paired $t$-test; $\mathrm{P}=0.023 ; \mathrm{N}=50$ for each group). However, no significant change was observed in IL-4, the most important Th2 cytokine. Analysis of ovarian cancer tissue and adjacent tissue lysates revealed that the expression of Th1 cytokines (namely IFN $\gamma$ and TNF $\alpha$ ) was significantly increased in the cancer tissues: IFN $\gamma$ was increased 4.67-fold (paired $t$-test; $\mathrm{P}$ $=0.011 ; \mathrm{N}=50$ for each group), and TNF $\alpha$ was increased 1.63 -fold (paired $t$-test; $\mathrm{P}=0.031 ; \mathrm{N}$ $=50$ for each group). IL- 6 , a Th 2 cytokine, was significantly decreased in cancer tissues $(0.07-$ fold; paired $t$-test; $\mathrm{P}=0.01 ; \mathrm{N}=50$ for each group). Thus, the immune system of the ovarian cancer patients was shifted toward Th1 cytokines compared with the healthy individuals.

\section{Differences in the ratios of various cytokines in ovarian cancer patients}

To investigate the diagnostic value of changes in the Th1-Th2 balance for ovarian cancer, serum TNF $\alpha / \mathrm{IL}-4$ and IFN $\gamma / \mathrm{IL}-4$ ratios (Tang et al., 2015) were used as indicators of the Th1-Th2 balance (Figure 2A and B). The TNF $\alpha /$ IL-4 and IFN $\gamma /$ IL-4 ratios were significantly higher in the sera of patients with ovarian cancer $(\mathrm{P}=0.0134)$. Receiver operating characteristic (ROC) curve analysis revealed that the TNF $\alpha / \mathrm{IL}-4$ ratio differed more than the IFN $\gamma / \mathrm{IL}-4$ ratio or the levels of IL-4, TNF $\alpha$, or IFN $\gamma$ between the healthy controls and the ovarian cancer patients. The area under the curve (AUC) values for the TNF $\alpha / \mathrm{IL}-4$ ratio, the IFN $\gamma / \mathrm{IL}-4$ ratio, IL-4, TNF $\alpha$, and IFN $\gamma$ were $0.846,0.803,0.314,0.831$, and 0.753, respectively (Figure 2C).

\section{Usefulness of the serum TNF $\alpha /$ IL-4 ratio for the prognostic evaluation of ovarian cancer treatment}

The 50 patients with ovarian cancer were classified into three groups according to the 
TNFa/IL-4 ratio (group 1, more than 5; group 2, 2-5; and group 3, less than 2), and changes in the level of cancer antigen 125 (CA125) were determined in each group after treatment. CA125 levels decreased significantly in all groups after ovarian cancer treatment, then gradually increased. In the 4th and 5th month after treatment, CA125 levels were significantly higher in groups 2 and 3 than in group 1, thus indicating their poor prognoses. Life-cycle curve analysis revealed that survival time was significantly longer in group 1 than in groups 2 and 3. Rank test analysis demonstrated that the TNF $\alpha / \mathrm{IL}-4$ ratio was significantly correlated with survival time ( $\mathrm{P}=0.021$; Figure 3$)$.
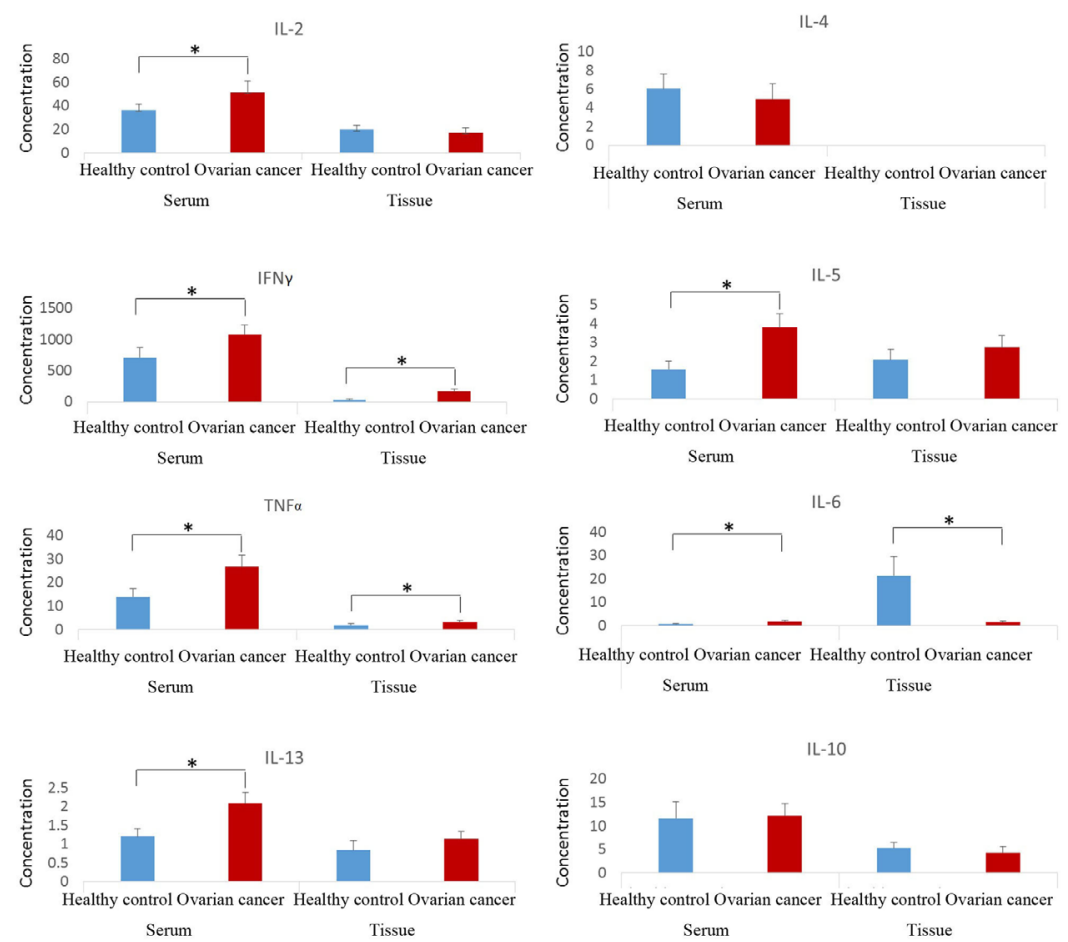

Figure 1. Levels of type 1 Thelper cell (Th1) factors (IL-2, IL-13, IFN $\gamma$, and TNF $\alpha$ ) and type 2 T helper cell (Th2) factors (IL-4, IL-5, IL-6, and IL-10) in the sera and cancer tissues of ovarian cancer patients compared with the healthy controls. The data are reported as means $\pm \mathrm{SE}$. $* \mathrm{P}<0.05$, paired $t$-test for serum samples and tissue samples $(\mathrm{N}=50$ for each group).

Table 1. Cytokine expression levels in type 1 and type $2 \mathrm{~T}$ helper cells (Th1 and Th2 cells) in the sera and cancer tissues of ovarian cancer patients.

\begin{tabular}{|c|c|c|c|c|c|c|}
\hline \multirow[t]{2}{*}{ Cytokine } & \multicolumn{2}{|c|}{ Serum $(\mathrm{pg} / \mathrm{mL})$} & \multirow[t]{2}{*}{$P$ value } & \multicolumn{2}{|c|}{ Tissue $(\mathrm{pg} / \mathrm{mL})$} & \multirow[t]{2}{*}{$\mathrm{P}$ value } \\
\hline & Control & Ovarian cancer & & Control & Ovarian cancer & \\
\hline IL-4 & $6.03 \pm 1.63$ & $4.92 \pm 1.63$ & 0.111 & & & \\
\hline IL-5 & $1.57 \pm 0.44$ & $3.79 \pm 0.75$ & 0.023 & $2.07 \pm 0.55$ & $2.74 \pm 0.62$ & 0.541 \\
\hline IL-6 & $0.69 \pm 0.21$ & $1.78 \pm 0.38$ & 0.014 & $21.28 \pm 8.38$ & $1.52 \pm 0.42$ & 0.010 \\
\hline IL-10 & $11.63 \pm 3.56$ & $12.17 \pm 2.55$ & 0.213 & $5.18 \pm 1.21$ & $4.29 \pm 1.34$ & 0.321 \\
\hline IL-2 & $36.21 \pm 5.17$ & $51.44 \pm 9.37$ & 0.023 & $19.55 \pm 3.67$ & $16.89 \pm 4.12$ & 0.432 \\
\hline IL-13 & $1.20 \pm 0.21$ & $2.08 \pm 0.29$ & 0.012 & $0.83 \pm 0.26$ & $1.14 \pm 0.20$ & 0.178 \\
\hline IFN $\gamma$ & $711.43 \pm 155.68$ & $1,080.81 \pm 151.58$ & 0.045 & $36.07 \pm 15.83$ & $168.16 \pm 43.16$ & 0.011 \\
\hline TNF $\alpha$ & $13.93 \pm 3.41$ & $26.66 \pm 4.86$ & 0.013 & $1.97 \pm 0.68$ & $3.21 \pm 0.65$ & 0.032 \\
\hline
\end{tabular}

Data are reported as median $\pm \mathrm{SE}$. 
A
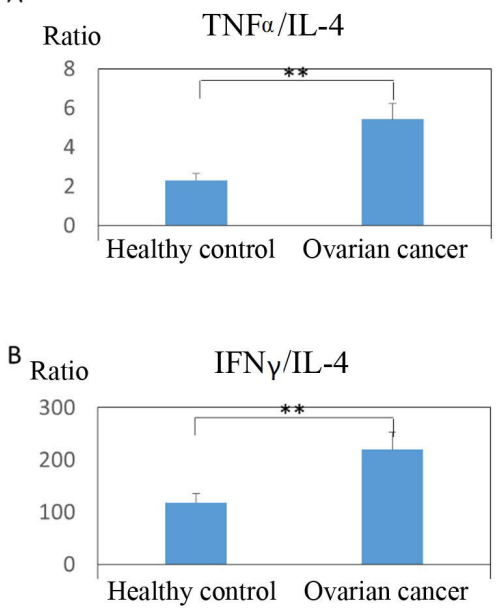

C

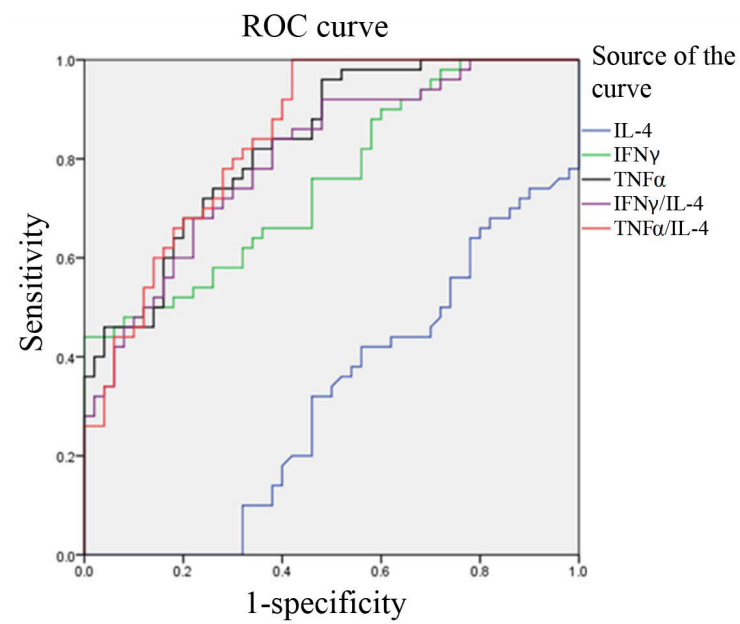

Figure 2. Alterations in the balance between type 1 and type $2 \mathrm{~T}$ helper cells (the Th1-Th2 balance) in the sera of ovarian cancer patients. A. and B. The TNF $\alpha /$ IL- 4 and IFN $\gamma / \mathrm{IL}-4$ ratios were significantly increased in the sera of ovarian cancer patients (paired $t$-test, $* * \mathrm{P}<0.01$, and $\mathrm{N}=50$ for each group). $\mathbf{C}$. The area under the curve (AUC) values for IL-4, IFN $\gamma$, TNF $\alpha$, the IFN $\gamma / \mathrm{IL}-4$ ratio, and the TNF $\alpha / \mathrm{IL}-4$ ratio for the diagnosis of ovarian cancer were $0.314,0.753,0.831,0.803$, and 0.846 , respectively, in receiver operating characteristic (ROC) curve analysis.

TNFa/IL-4 ratio (group 1, more than 5; group 2, 2-5; and group 3, less than 2), and changes in the level of cancer antigen 125 (CA125) were determined in each group after treatment. CA125 levels decreased significantly in all groups after ovarian cancer treatment, then gradually increased. In the 4th and 5th month after treatment, CA125 levels were significantly higher in groups 2 and 3 than in group 1, thus indicating their poor prognoses. Life-cycle curve analysis revealed that survival time was significantly longer in group 1 than in groups 2 and 3. Rank test analysis demonstrated that the TNF $\alpha /$ IL-4 ratio was significantly correlated with survival time $(\mathrm{P}=0.021$; Figure 3$)$.
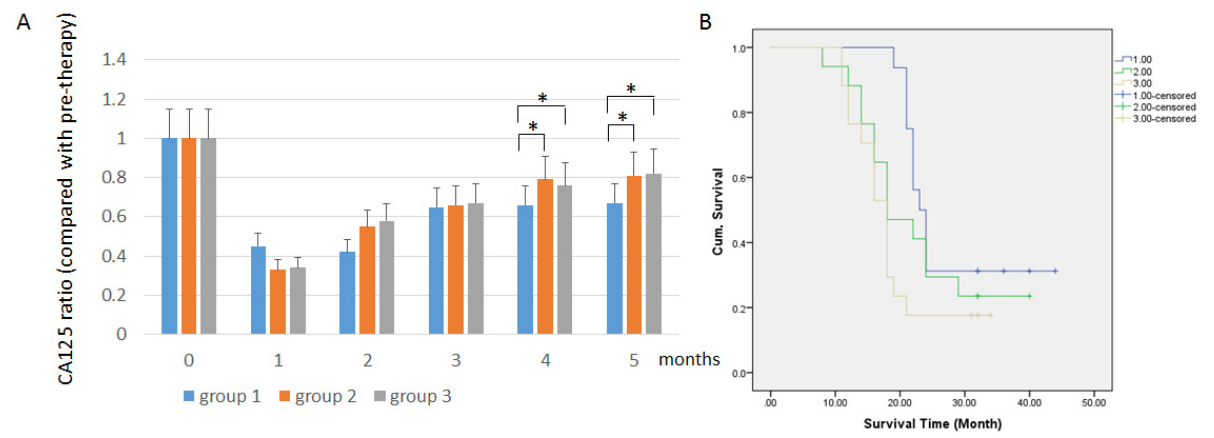

Figure 3. TNF $\alpha / \mathrm{IL}-4$ ratio was positively correlated with the prognosis of ovarian cancer patients. A. Cancer antigen 125 (CA125) levels changed after ovarian cancer treatment. All CA125 levels had decreased significantly 1 month after treatment, and then they gradually increased. In the 4th and 5th month after treatment, the CA125 levels in groups 2 and 3 were significantly higher than that in group 1. B. Life-cycle curve analysis revealed that the survival time in group 1 was significantly longer than that in groups 2 and 3. 


\section{DISCUSSION}

The Th1-Th2 balance has been reported to play an important role in the development and progression of many cancers, but its role in ovarian cancer remains unclear. In this study, Th1-Th2 levels were quantified in the sera and cancer tissues of patients with ovarian cancer before treatment using antibody arrays. Th1 cytokines (IL-2, TNF $\alpha$, IFN $\gamma$, and IL-13) were significantly increased in the sera and cancer tissues of the ovarian cancer patients. Of the Th2 cytokines, only IL-6 and IL-5 were increased in cancer tissues, and IL-6 was significantly decreased. Compared with the healthy controls, IFN $\gamma / \mathrm{IL}-4$ and TNF $\alpha / \mathrm{IL}-4$ ratios were significantly increased in the peripheral blood of the ovarian cancer patients, indicating that the Th1-Th2 balance was shifted toward Th1, representing an anti-tumor shift. These results also indirectly demonstrate that an altered Th1-Th2 balance might be caused by ovarian cancer rather than the reverse relationship. According to traditional theory, shifting the Th1-Th2 balance toward Th2 enables tumor cells to avoid immune surveillance, leading to tumorigenesis (Clerici et al., 1996). However, we observed that the Th1-Th2 balance was shifted toward antitumor Th1 before treatment in the ovarian cancer patients. We also observed that IL- 6 was highly expressed in cancer-adjacent tissues but was significantly decreased in ovarian cancer tissues compared with adjacent tissues, consistent with the mRNA data (Kusuda et al., 2005). Therefore, IL-6 might be secreted by cancer-adjacent tissues and released into the blood.

This study also indicated that TNF $\alpha$, IFN $\gamma$, and the TNF $\alpha / \mathrm{IL}-4$ and IFN $\gamma / \mathrm{IL}-4$ ratios are useful for the diagnosis of ovarian cancer. The TNF $\alpha / \mathrm{IL}-4$ ratio provided better discriminating power than the use of TNF $\alpha$ or IFN $\gamma$ alone. ROC curve analysis demonstrated that the TNF $\alpha /$ IL-4 ratio differed more significantly than the IFN $\gamma / \mathrm{IL}-4$ ratio or the levels of IL-4, TNF $\alpha$, or IFN $\gamma$ between the healthy controls and the ovarian cancer patients. The AUC values were $0.846,0.803,0.314,0.831$, and 0.753 for the TNF $\alpha / \mathrm{IL}-4$ ratio, the IFN $\gamma / \mathrm{IL}-4$ ratio, IL-4, TNF $\alpha$, and IFN $\gamma$, respectively. However, because TNF $\alpha$, IFN $\gamma$, IL-4, and other factors are influenced by a variety of diseases, their specificity in the detection of ovarian cancer should be verified.

Additionally, we determined that the TNF $\alpha / \mathrm{IL}-4$ ratio was positively correlated with the prognosis of ovarian cancer; higher TNF $\alpha / \mathrm{IL}-4$ ratios indicated longer patient survival. This result is consistent with previous findings that a high Th1-Th2 ratio in tumors predicts good prognosis in gastric and liver cancers (Nagai et al., 2008; Chang et al., 2014). However, owing to the inconsistencies regarding clinical stage and patient age, further experiments are required to confirm the relevance of ovarian cancer staging and age to the Th1-Th2 balance.

This study had several limitations. The sample size was small. Therefore, studies with a larger sample size are needed to confirm the results of this study. Although the rank test demonstrated that the TNF $\alpha /$ IL-4 ratio was positively correlated with prognosis, additional clinical samples are needed for verification. Second, the clinical stage was inconsistent. All 50 ovarian cancer cases studied here ranged from stage 1 to stage 3 . Third, the patients differed in age. Although the patients and healthy controls were age-matched, their ages ranged from 40 to 65 years. The influence of the factors mentioned above on Th1-Th2 balance requires further validation. In future studies, we will increase the sample size and refine the clinical information to further clarify the effect of the Th1-Th2 balance on ovarian cancer.

This study is the first to reveal a correlation between Th1-Th2 balance and the diagnosis and prognosis of ovarian cancer; in particular, the TNF $\alpha / \mathrm{IL}-4$ ratio was identified as relevant for the diagnosis and prognostic evaluation of ovarian cancer. Thus, the Th1-Th2 balance represents a valuable tool for use in clinical practice. 


\title{
Conflicts of interest
}

The authors declare no conflict of interest.

\section{ACKNOWLEDGMENTS}

\author{
Research supported by the National Natural Science Funds for Young Scholars in \\ China (\#81101701).
}

\section{REFERENCES}

Aoki Y, Tsuneki I, Sasaki M, Watanabe M, et al. (2000). Analysis of TH1 and TH2 cells by intracellular cytokine detection with flow cytometry in patients with ovarian cancer. Gynecol. Obstet. Invest. 50: 207-211. http://dx.doi.org/10.1159/000010312

Block MS, Nevala WK, Leontovich AA and Markovic SN (2011). Differential response of human and mouse dendritic cells to VEGF determines interspecies discrepancies in tumor-mediated TH1/TH2 polarity shift. Clin. Cancer Res. 17: 1776-1783. http://dx.doi.org/10.1158/1078-0432.CCR-10-2836

Chang WJ, Du Y, Zhao X, Ma LY, et al. (2014). Inflammation-related factors predicting prognosis of gastric cancer. World J. Gastroenterol. 20: 4586-4596. http://dx.doi.org/10.3748/wjg.v20.i16.4586

Chen M, Hao X, Zhang Y, Lu B, et al. (2004). The clinical significance of detection of Th1/Th2 cell cytokines in lung cancer. Zhongguo Fei Ai Za Zhi 7: 214-217.

Chornokur G, Amankwah EK, Schildkraut JM and Phelan CM (2013). Global ovarian cancer health disparities. Gynecol. Oncol. 129: 258-264. http://dx.doi.org/10.1016/j.ygyno.2012.12.016

Clerici M, Clerici E and Shearer GM (1996). The tumor enhancement phenomenon: reinterpretation from a Th1/Th2 perspective. J. Natl. Cancer Inst. 88: 461-462. http://dx.doi.org/10.1093/jnci/88.7.461

Green VL, Alexandropoulou A, Walker MB, Walker AA, et al. (2010). Alterations in the Th1/Th2 balance in breast cancer patients using reflexology and scalp massage. Exp. Ther. Med. 1: 97-108.

Höhn AK, Einenkel J, Wittekind C and Horn LC (2014). New FIGO classification of ovarian, fallopian tube and primary peritoneal cancer. Pathologe 35: 322-326. http://dx.doi.org/10.1007/s00292-014-1908-0

Jones EA, Pringle JH, Angel CA and Rees RC (2002). Th1/Th2 cytokine expression and its relationship with tumor growth in B cell non-Hodgkin's lymphoma (NHL). Leuk. Lymphoma 43: 1313-1321. http://dx.doi. org $/ 10.1080 / 10428190290026385$

Kidd P (2003). Th1/Th2 balance: the hypothesis, its limitations, and implications for health and disease. Altern. Med. Rev. 8: 223-246.

Kusuda T, Shigemasa K, Arihiro K, Fujii T, et al. (2005). Relative expression levels of Th1 and Th2 cytokine mRNA are independent prognostic factors in patients with ovarian cancer. Oncol. Rep. 13: 1153-1158.

Mills KH, Barnard A, Watkins J and Redhead K (1993). Cell-mediated immunity to Bordetella pertussis: role of Th1 cells in bacterial clearance in a murine respiratory infection model. Infect. Immun. 61: 399-410.

Nagai H, Miyaki D, Matsui T, Kanayama M, et al. (2008). Th1/Th2 balance: an important indicator of efficacy for intraarterial chemotherapy. Cancer Chemother. Pharmacol. 62: 959-963. http://dx.doi.org/10.1007/s00280-008-0685-y

Shurin MR, Lu L, Kalinski P, Stewart-Akers AM, et al. (1999). Th1/Th2 balance in cancer, transplantation and pregnancy. Springer Semin. Immunopathol. 21: 339-359. http://dx.doi.org/10.1007/BF00812261

Sugiyama T and Kou T (2008). Classification and treatment of ovarian cancer. Gan To Kagaku Ryoho 35: 228-232.

Tang F, Wang F, An L and Wang X (2015). Upregulation of Tim-3 on CD4(+) T cells is associated with Th1/Th2 imbalance in patients with allergic asthma. Int. J. Clin. Exp. Med. 8: 3809-3816.

Wada T, Iijima H, Sekine F, Kato M, et al. (2003). Effect of PSK on Th1/Th2 balance in tumor-bearing mice. Gan To Kagaku Ryoho 30: 1798-1801.

Zhang L, Hao CQ, Miao L and Dou XG (2014). Role of Th1/Th2 cytokines in serum on the pathogenesis of chronic hepatitis C and the outcome of interferon therapy. Genet. Mol. Res. 13: 9747-9755. http://dx.doi.org/10.4238/2014.November.27.2

Zhao WH, Li L, Zhang B, Zhang WD, et al. (2008). Enhancement of CD4+ T cell activities and modulation of Th1/Th2 lineage development in radiated tumor-bearing rats treated with male zooid of Antheraea pernyi extracts. World $J$. Gastroenterol. 14: 2094-2099. http://dx.doi.org/10.3748/wjg.14.2094

Zhu X, Ying LS, Xu SH, Zhu CH, et al. (2010). Clinicopathologic and prognostic significance of serum levels of cytokines in patients with advanced serous ovarian cancer prior to surgery. Zhonghua Bing Li Xue Za Zhi 39: 666-670. 